\title{
Polyphosphazene-Based Drug Self-Framed Delivery System as a Universal Intelligent Platform for Combination Therapy against Multidrug-Resistant Tumors
}

Shenglei Hou ${ }^{\dagger}$, Shiliu Zhou ${ }^{\dagger}$, Shuangshuang Chen*, and Qinghua Lu**, ${ }^{*}$

${ }^{\dagger}$ School of Chemistry and Chemical Engineering, The State Key Laboratory of Metal Matrix Composites, Shanghai Jiao Tong University, Shanghai, 200240, China

$\$$ School of Chemical Science and Engineering, Tongji University, Shanghai, 200092, China

*Email:Email: fjchenss@sjtu.edu.cn.

** Email:Email: qhlu@sjtu.edu.cn.

KEYWORDS: combination therapy, synergistic effect, drug self-framed delivery system, MDR cancer, polyphosphazene 
-Section A: the materials and experiment detail

-Section B: the finger and caption.

-Figure S1: SEM images of four different kinds of DSFDS;

- Figure S2: Size distribution and UV-vis spectra of three different kinds of DSFDS;

-Figure S3: Photographs of different DSFDSs dispersed in PBS for $72 \mathrm{~h}$;

- Figure S4: FTIR spectra and EDX spectrum of three different kinds of DSFDS;

•Figure S5: The character of DOX-RES-CysM-CPPZ NPs;

-Figure S6: The standard curves of UV absorbance of free DOX and free RES;

-Figure S7: DLS data of DOX-RES-CysM-CPPZ NPs during degradation processes;

-Figure S8: TEM images of a HeLa cell;

- Table S1: The $\mathrm{IC}_{50}(\mu \mathrm{M})$ values of different treatment after incubation with HeLa or $\mathrm{HeLa} / \mathrm{ADR}$ cells for $24 \mathrm{~h}$. 


\section{MATERIALS AND METHODS}

\section{Materials:}

(H-Cys-OMe) $)_{2} \cdot 2 \mathrm{HCl}$ was obtained from GL Biochem (Shanghai) Co. Ltd. Hexachlorocyclotriphosphazene (HCCP) was obtained from Tokyo Chemical Industry (Japan) Co., Ltd. Resveratrol (RES), doxorubicin (DOX), acetonitrile $(\mathrm{MeCN})$, triethylamine (TEA), and glutathione (GSH) were purchased from Adamas-Beta (China). The Annexin V-FITC/PI Cell Apoptosis Kit and LysoTracker Green were provided by Thermo Fisher Scientific (Shanghai, China). N-ethylmaleimide (NEM, a thiol scavenging agent) was provided by Shanghai Macklin Biochemical Co. Ltd. Fetal bovine serum (FBS), Dulbecco's Modified Eagle Medium with High Glucose (DMEM) was provided by Gibco (USA). Penicillin/streptomycin, anti-P-gp monoclonal antibody and secondary antibody conjugated with FITC, and the Cell Counting Kit-8 (CCK-8) were provided by Beyotime Biotechnology Co. Ltd (China). Milli-Q water (Millipore) was used in all experiments. L929 cells and HeLa cells were provided by the Shanghai Institute of Biochemistry and Cell Biology, Chinese Academy of Science. The human DOX-resistant cervical cancer cell line, HeLa/ADR, was provided by the Shanghai Bioleaf Biotech Co., Ltd. Female BALB/c nude mice (4-5 weeks old) were provided by Shanghai SLAC Laboratory Animal Co. Ltd.

Syntheses of DOX-CPPZ，DOX-CysM-CPPZ，DOX-RES-CysM-CPPZ, DOX-TPP-CysM-CPPZ, DOX-Cur-CysM-CPPZ and DOX-Mit-CysM-CPPZ NPs. 
DOX-CPPZ NPs were synthesized using the method previously published by our goup. ${ }^{1}$

HCCP-CysM oligomer was firstly synthesized using the previously published method. ${ }^{2,3}$ HCCP-CysM-RES oligomer was synthesized as follows: HCCP-CysM oligomer (0.5 mL), RES (25 mg), and TEA ( $2 \mathrm{~mL}$, used as acid-binding agent) were added to $\mathrm{MeCN}$ (40 mL). The mixture was subjected to ultrasonic irradiation at ambient temperature for $8 \mathrm{~h}$ and then the supernatant was collected after centrifugation.

DOX-RES-CysM-CPPZ NPs were synthesized as follows: TEA (3 mL) and DOX $30 \mathrm{mg}$ ) were dissolved in HCCP-CysM-RES oligomer $(40 \mathrm{~mL})$. The mixture was subjected to ultrasonic irradiation at ambient temperature for $8 \mathrm{~h}$. The resulting product was collected by centrifugation and washed successively with Milli-Q water $(10 \mathrm{~mL})$ and ethyl alcohol $(4 \times 10 \mathrm{~mL})$. The product was dried under vacuum at ambient temperature. DOX-TPP-CysM-CPPZ, DOX-Cur-CysM-CPPZ and DOX-Mit-CysM-CPPZ NPs were synthesized in a similar manner to that of DOX-RES-CysM-CPPZ NPs.

The amount of unreacted DOX in the reaction was determined by comparing the UV-vis absorbance of the supernatant at $480 \mathrm{~nm}$ with the standard curve of DOX solution at different concentrations under the same conditions. The drug-loading rate of the DSFDS was calculated by subtracting the amount of unreacted DOX from the total used in the reaction. The drug-loading rate formula is: $\mathrm{R}_{\mathrm{DOX}-\mathrm{loaded}}=\left[\left(\mathrm{M}_{\mathrm{DOX}}-\right.\right.$ $\left.\mathrm{M}_{\text {un-DOX }} / \mathrm{M}_{\mathrm{DSFDS}}\right] \times 100 \%$, where $\mathrm{M}_{\mathrm{DSFDS}}$ is the total mass of DSFDS NPs, $\mathrm{M}_{\mathrm{DOx}}$ is 
the total mass of DOX used in the reaction, and $\mathrm{M}_{\mathrm{un}-\mathrm{DOX}}$ is the mass of unreacted DOX. The RES loading rate of DSFDS NPs was calculated in a similar manner.

\section{Characterization}

Transmission electron microscopy (TEM, Japan, JEOL JEM-2100, $200 \mathrm{kV}$ ) and Field emission scanning electron microscopy (FE SEM, Netherlands, Philips Sirion $200,20 \mathrm{kV}$ ) were used to characterize the morphologies of the resultant DSFDS. Image $\mathrm{J}$ (v1.41, $n$ Light, USA) was used to determine the size and distribution of the prepared nanoparticles from TEM or SEM micrographs by image analysis. A digital camera (IXUS 800IS, Canon) was used to collect photographs. A Malvern ZetaSizer Nano ZS was used for dynamic light scattering (DLS) measurements using a laser at 633 nm. A PE Lambda 35 spectrophotometer was used to record ultraviolet-visible (UV-vis) absorption spectra. Fourier-transform infrared (FT-IR) spectra were recorded on a Paragon 1000 (PerkinElmer) spectrometer. Fluorescence spectra were recorded on a PerkinElmer LS 50B fluorescence spectrometer. A TCS SP8 STED 3× super-resolution multiphoton confocal microscope (Leica, He-Ne and Ar lasers) was used to collect fluorescence images of cells. Bruker AVANCE III $400 \mathrm{MHz}$ and 600 $\mathrm{MHz}$ (Germany) spectrometers were used to record the ${ }^{31} \mathrm{P}$ NMR spectra, with chemical shifts quoted relative to $\mathrm{K}_{2} \mathrm{HPO}_{3}$.

\section{In vitro Drug Release}

In vitro drug release was monitored by dialysis. DOX-RES-CysM-CPPZ NPs (10 mg) was placed into dialysis tubes $(1 \mathrm{KD})$ and the tubes stirred in phosphate-buffered 
saline solution (PBS, $100 \mathrm{~mL}, \mathrm{pH} 7.4,6.5$ or 5.5 with or without $10 \mu \mathrm{M} \mathrm{GSH}$ ) at $37^{\circ} \mathrm{C}$. 
Standard curves of free DOX UV absorbance at $480 \mathrm{~nm}$ were obtained in PBS solutions at different concentrations (Figure S3). The dialyzates were collected at different time points and the concentration of released DOX was calculated from the UV absorbance at $480 \mathrm{~nm}$ compared to the standard curves. The RES in vitro release curves were determined in a similar manner.

\section{Tumor Cell Culture}

HeLa and HeLa/ADR cells were used for the cell experiments. Cells were cultured under a humidified atmosphere of $5 \% \mathrm{CO}_{2}$ at $37^{\circ} \mathrm{C}$. DMEM was supplemented with $10 \%$ FBS and $1 \%$ penicillin/streptomycin.

\section{In vitro Uptake}

A four-chamber glass bottom dish was used to seed HeLa and HeLa/ADR cells and incubated overnight. Cells were cultured with medium containing DOX-RES-CysM-CPPZ NPs for $2 \mathrm{~h}$ and then the cells were washed three times with PBS. The cells were incubated with fresh DMEM for a further 1, 3, 6 and $12 \mathrm{~h}$. The cells were co-incubated with DMEM supplemented with LysoTracker Green for staining. After incubation for $1 \mathrm{~h}$, the cells were washed twice with cold PBS, fixed with paraformaldehyde (4 wt $\%$ ) for $30 \mathrm{~min}$, and then washed twice with cold PBS. Confocal laser scanning microscopy was used to image the fixed cells.

Immunofluorescence was used to observe the difference between entry of free DOX or DOX- RES-CysM-CPPZ NPs into HeLa and HeLa/ADR cells. Cells were seeded in 6-well culture plates for overnight incubation, treated with free RES, free DOX, DOX-CysM-CPPZ NPs or DOX-RES-CysM-CPPZ NPs for $6 \mathrm{~h}$, and then 
washed twice with cold PBS. Cells were co-incubated with DMEM supplemented with LysoTracker Green for staining. After incubation for $1 \mathrm{~h}$, the cells were washed twice with cold PBS, fixed with paraformaldehyde (4 wt\%) for $30 \mathrm{~min}$, and then washed twice with cold PBS. Finally, the fixed cells were stained with $0.1 \mu \mathrm{g} \mathrm{mL}{ }^{-1}$ DAPI solution for $15 \mathrm{~min}$ and then washed twice with PBS. Fluorescence images of the fixed cells were collected by confocal laser scanning microscopy.

For immunofluorescence staining of P-glycoprotein, HeLa/ADR cells were seeded in four-chamber glass bottom dishes for overnight incubation. The cells were treated with free DOX, free RES, DOX-CysM-CPPZ NPs or DOX-RES-CysM-CPPZ NPs for $48 \mathrm{~h}$, fixed with paraformaldehyde ( $4 \mathrm{wt} \%$ ) for $30 \mathrm{~min}$, washed three times with cold PBS for $5 \mathrm{~min}$, and finally permeabilized using Triton X-100 (0.1\%) in PBS for $10 \mathrm{~min}$. Fixed cells were pre-incubated at ambient temperature for $30 \mathrm{~min}$ in PBS containing 5\% BSA. The cells were treated with primary antibody (anti-P-gp monoclonal antibody, 1:200 dilution) for $1 \mathrm{~h}$ at ambient temperature and then incubated with FITC-conjugated secondary antibody. DAPI was added to the secondary antibody mixture to visualize nuclei. Confocal laser scanning microscopy was used to collect and analyze fluorescence images.

\section{Cell Viability Test}

A 96-well culture plate was used to incubate HeLa and HeLa/ADR cells $\left(10^{5}\right.$ cells $\mathrm{mL}^{-1}, 100 \mu \mathrm{L}$ of medium). After overnight incubation, free DOX, free RES, DOX-CysM-CPPZ NPs or DOX-RES-CysM-CPPZ NPs (at equivalent drug concentrations of $0,0.001,0.005,0.1,0.5,1,5,10,50,100$, and $500 \mu \mathrm{M}$ in culture 
medium without FBS) were co-incubated with cells for $4 \mathrm{~h}$. The cells were incubated with fresh media for a further $24 \mathrm{~h}$ and then each well was treated with WST-8 reagent $(10 \mu \mathrm{L})$. After incubation for a further $2 \mathrm{~h}$, a microplate reader (Bio-Rad, model 680) was used to record the absorbance at $450 \mathrm{~nm}$. Cell viability was calculated as the percentage absorbance of the sample well compared to that of the controls.

To study the influence of intracellular GSH on DOX-RES-CysM-CPPZ NPs mediated cell death, HeLa/ADR cells were incubated overnight with or without NEM for 2 h. The cells were then washed twice with cold PBS. Free RES, free DOX, DOX-CysM-CPPZ NPs and DOX-RES-CysM-CPPZ NPs (at an equivalent drug concentration of $5 \mu \mathrm{M}$ in culture medium without FBS) were added and the cells incubated for a further $24 \mathrm{~h}$. The viability of cells was determined using the WST-8 assay.

The cytotoxicity of each treatment was also evaluated by quantitative analysis of HeLa cell apoptosis. HeLa cells $\left(5 \times 10^{4}\right.$ cells per well) were seeded into a 24 -well plate. The cells were treated with PBS, free DOX, free RES, DOX-CysM-CPPZ NPs or DOX-RES-CysM-CPPZ NPs (at an equivalent drug concentration of $5 \mu \mathrm{M}$ ) and incubated for 2 days. Flow cytometry was used to measure and quantify apoptosis using an Annexin V-FITC/PI Apoptosis Detection Kit.

\section{In Vivo Antitumor Activity}

All animal experiments were conducted in strict observance of the guide for the care and use of laboratory animals by Shanghai Jiao Tong University Animal Study 
Committee. HeLa cells $\left(10^{7}\right)$ were implanted into the flank region of the mice by subcutaneous injection. The tumor volume was calculated using the following formula: Volume $=($ Tumor Length $) \times(\text { Tumor Width })^{2} / 2$. The volume is presented as relative tumor volume $\left(\mathrm{V}_{\mathrm{t}} / \mathrm{V}_{0}\right)$, where $\mathrm{V}_{0}$ is the average tumor volume on Day 0 and $\mathrm{V}_{\mathrm{t}}$ is the tumor volume at a given time.

In vivo imaging and biodistribution: The tumor-bearing mice were randomly divided into three groups of five. The mice were injected with PBS (120 $\mu \mathrm{L})$, DOX-RES-CysM-CPPZ NPs, or free DOX via the tail vein, at an equivalent DOX concentration of $0.17 \mathrm{mg} \mathrm{mL}^{-1}$. After injection, the in vivo red fluorescence signal of free DOX at different time points was recorded using an ex/in vivo imaging system with a 488/590 $\mathrm{nm}$ filter setting. The mice were sacrificed $24 \mathrm{~h}$ after injection and the key organs and tumors were excised for imaging and biodistribution analysis. The DOX fluorescence signal was obtained using the $488 / 590 \mathrm{~nm}$ filter setting.

In vivo tumor growth inhibition: When the HeLa tumor volumes reached 150-200 $\mathrm{mm}^{3}$, the mice were randomly divided into six groups of five and dosed intravenously with PBS $(100 \mu \mathrm{L})$, RES-CysM-CPPZ NPs, free DOX, free RES, DOX-CysM-CPPZ NPs, and DOX-RES-CysM-CPPZ NPs at an equivalent DOX concentration of 0.17 $\mathrm{mg} \mathrm{mL} \mathrm{m}^{-1}$ via the tail vein. Each treatment was carried out at six times at a time interval of 3 days. Body weights and tumor volumes of each mouse were regularly recorded. The mice were sacrificed 27 days after the first administration. The key organs and tumors were collected and fixed for H\&E or TUNEL assay, respectively. 
Statistical analysis: Statistical significance was determined by using one-way ANOVA analysis. NS $\mathrm{p}>0.05,{ }^{*} \mathrm{p}<0.05,{ }^{* *} \mathrm{p}<0.01,{ }^{* * *} \mathrm{p}<0.001,{ }^{* * * * *} \mathrm{p}<0.0001$. Data were expressed as mean $\pm \mathrm{SD}$.

\section{FIGURES AND CAPTION SECTIONS}
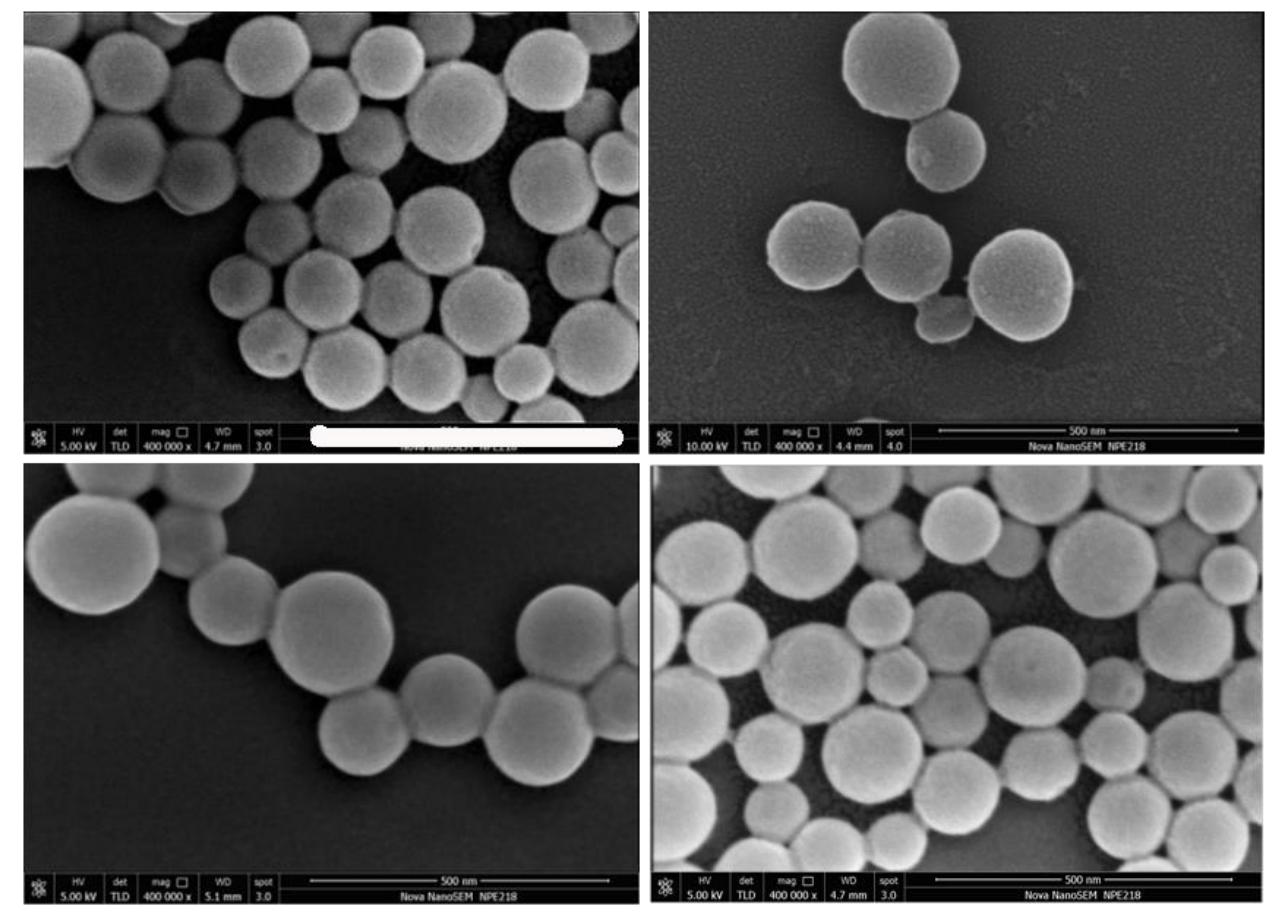

Figure S1. SEM images of DOX-RES-CysM-CPPZ NPs, DOX-Mit-CysM-CPPZ NPs, DOX-Cur-CysM-CPPZ NPs and DOX-TPP-CysM-CPPZ NPs. The scale bar is $500 \mathrm{~nm}$. 

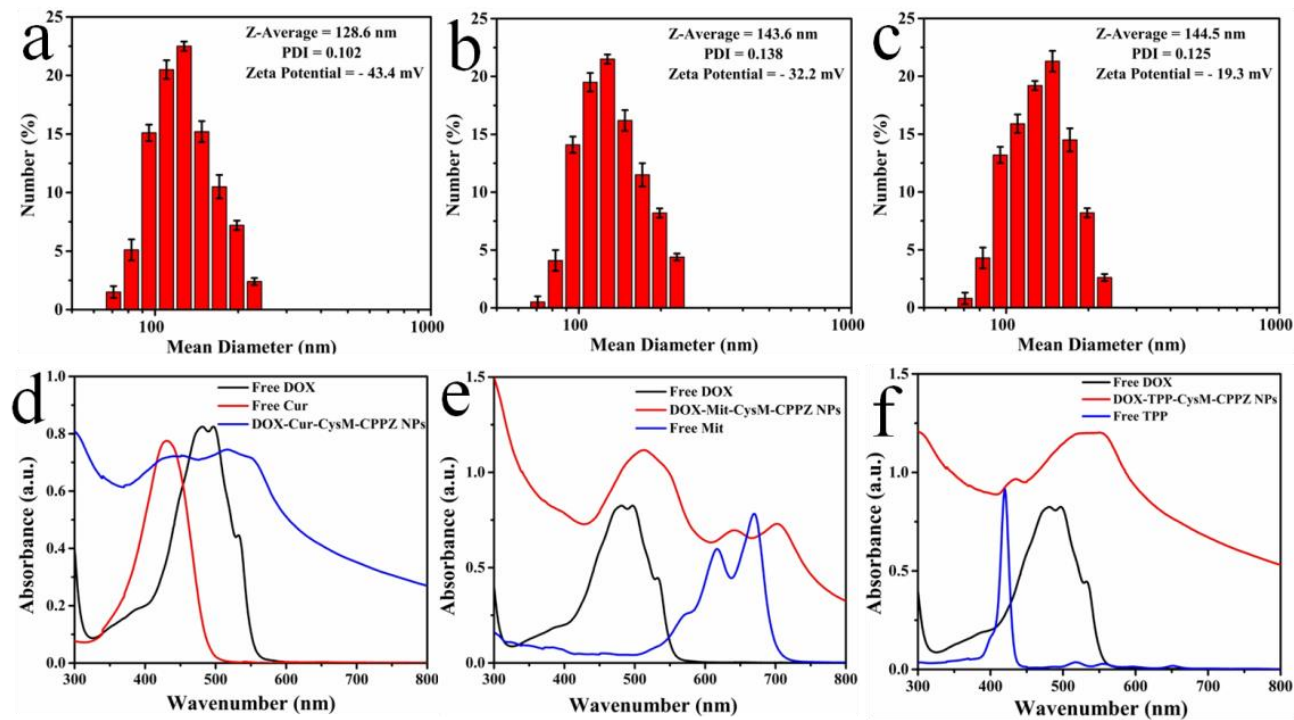

Figure S2. Size distribution (a-c) and UV-vis spectra (d-f) of DOX-Cur-CysM-CPPZ, DOX-Mit-CysM-CPPZ and DOX-TPP-CysM-CPPZ NPs.

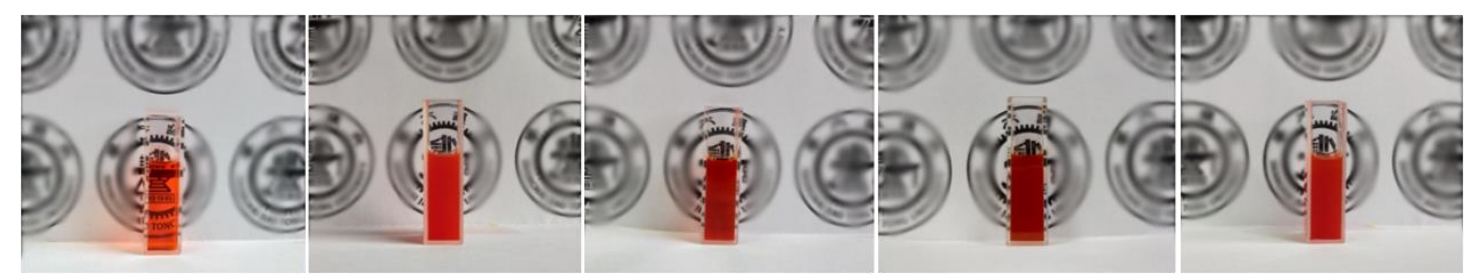

Figure S3. Photographs of free DOX, DOX-RES-CysM-CPPZ NPs, DOX-TPP-CysM-CPPZ, DOX-Cur-CysM-CPPZ and DOX-Mit-CysM-CPPZ NPs dispersed in PBS for $72 \mathrm{~h}$. 

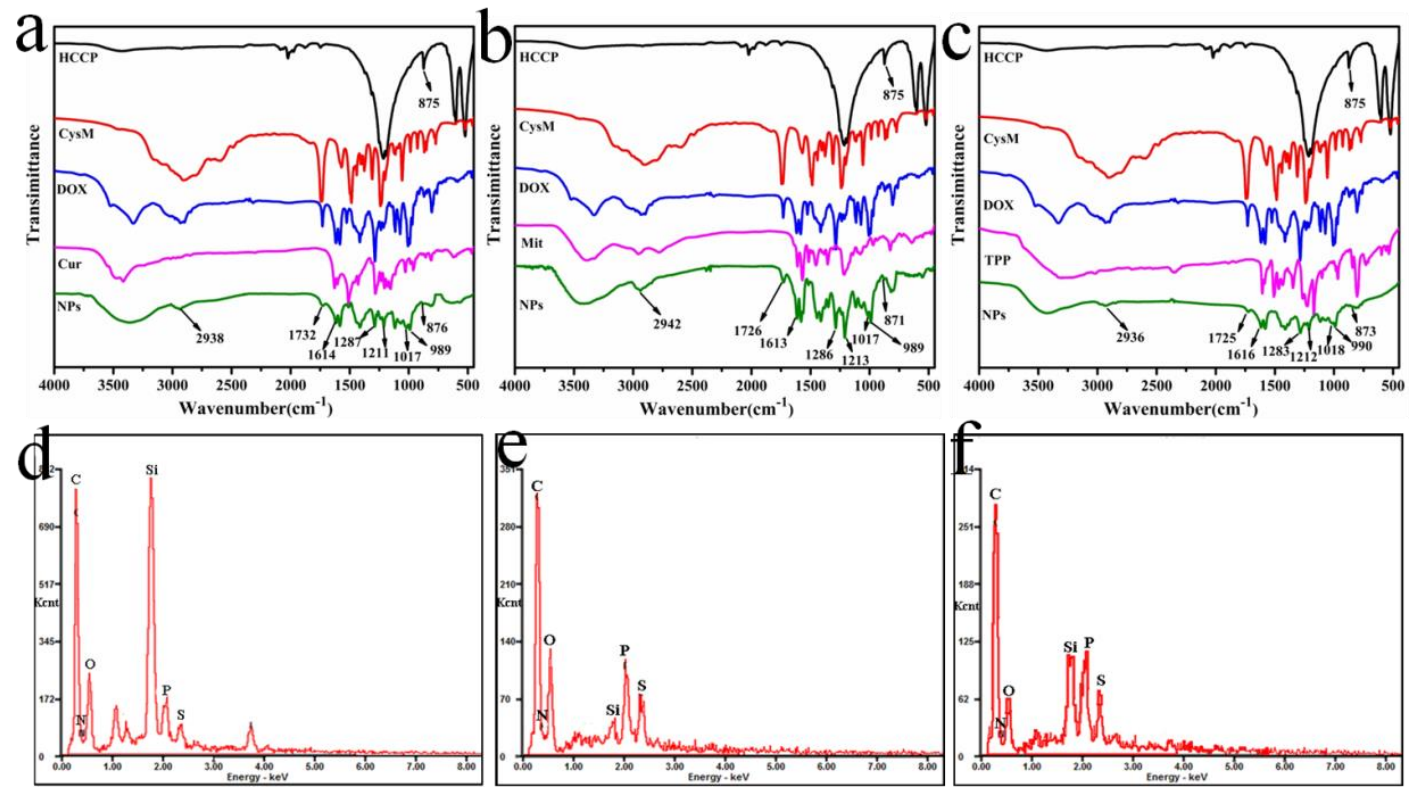

Figure S4. (a-c) FTIR spectra and (d-f) EDX spectrum of DOX-Cur-CysM-CPPZ, DOX-Mit-CysM-CPPZ and DOX-TPP-CysM-CPPZ NPs. 

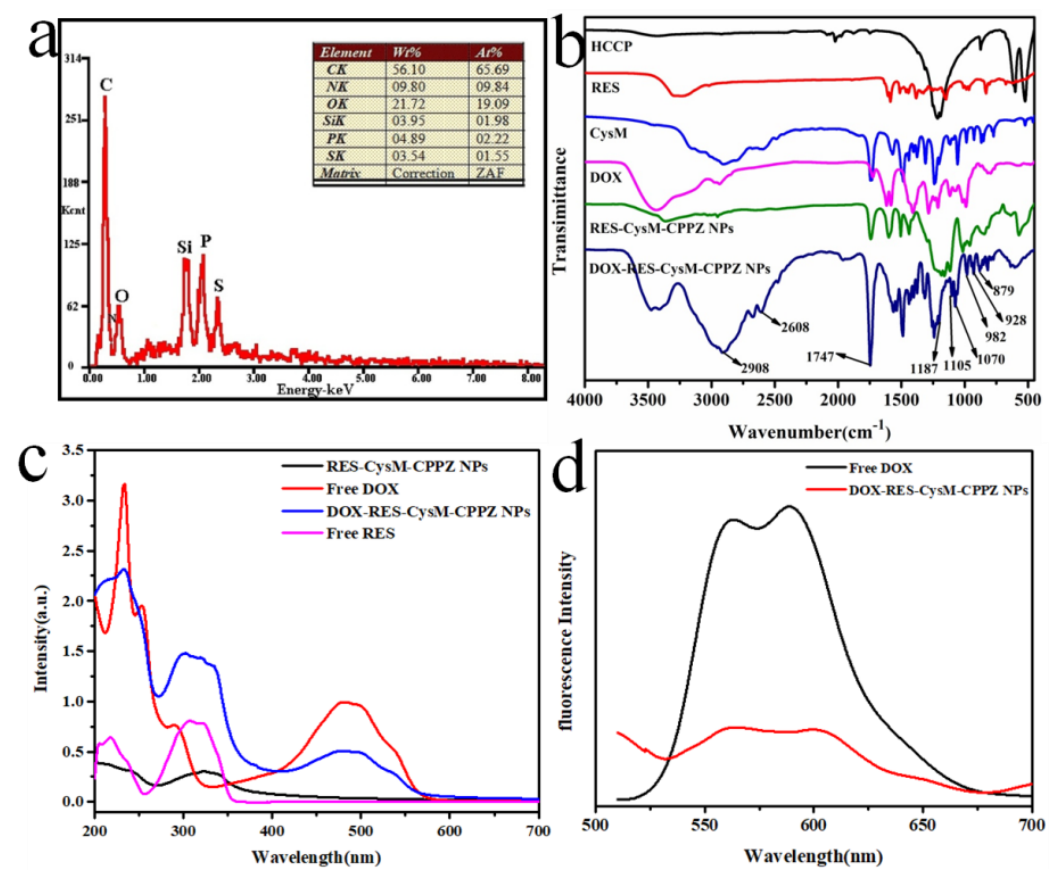

Figure S5. (a) EDX spectrum and elemental composition of DOX-RES-CysM-CPPZ

NPs. (b) FTIR spectra of free DOX, free RES, HCCP, RES-CysM-CPPZ NPS and DOX-RES-CysM-CPPZ NPs. (c) UV spectra of free DOX, free RES, RES-CysM-CPPZ NPS and DOX-RES-CysM-CPPZ NPs. (d) Fluorescence spectra of free DOX and DOX-RES-CysM-CPPZ NPs.
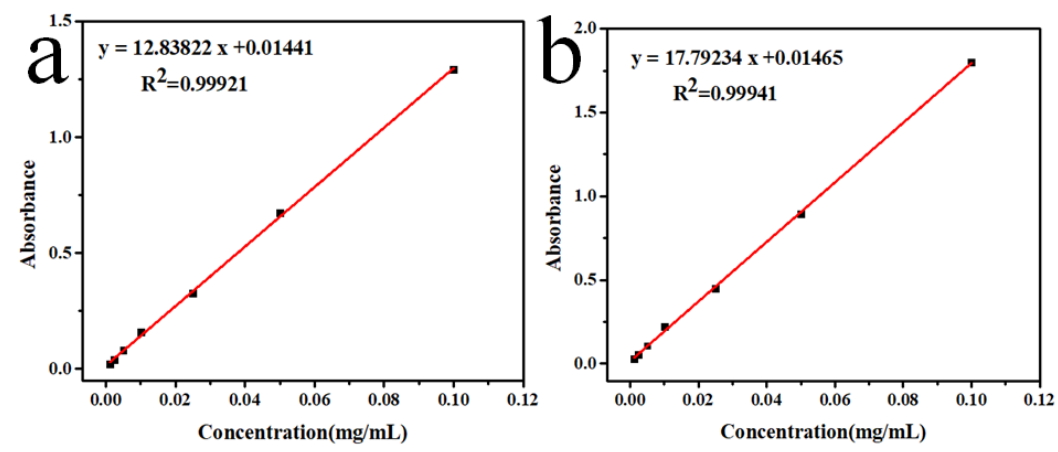

Figure S6. Standard curves of UV absorbance of (a) RES at $330 \mathrm{~nm}$ and (b) DOX at $480 \mathrm{~nm}$ versus concentration. 

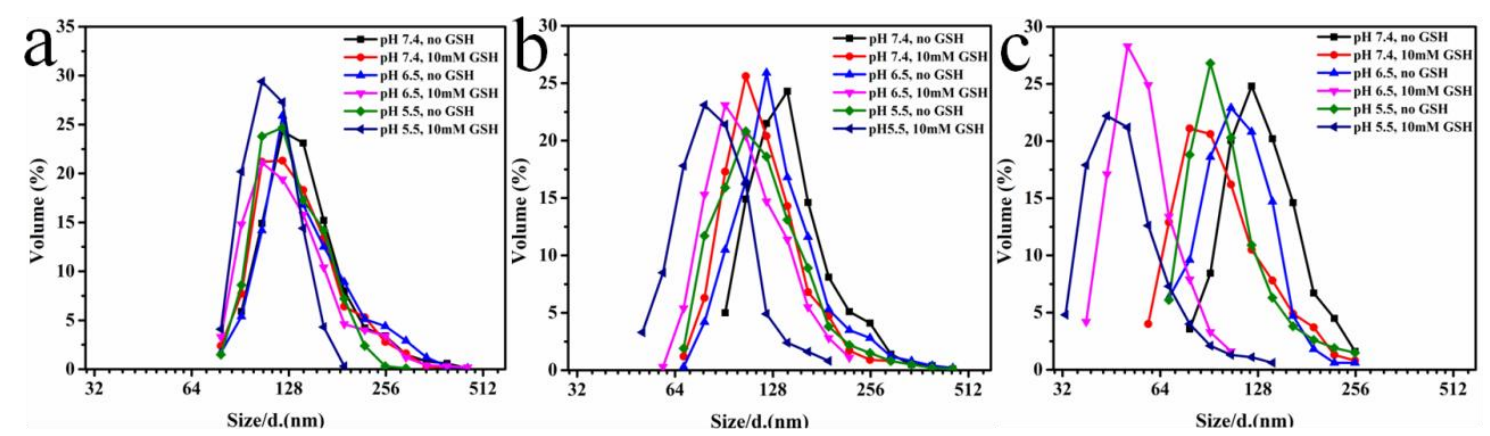

Figure S7. DLS data of DOX-RES-CysM-CPPZ NPs during degradation in PBS at $\mathrm{pH} 7.4,6.5$ and 5.5 with or without $10 \mathrm{mM} \mathrm{GSH}$ after (a) 1 day, (b) 2 days, and (c) 3 days.
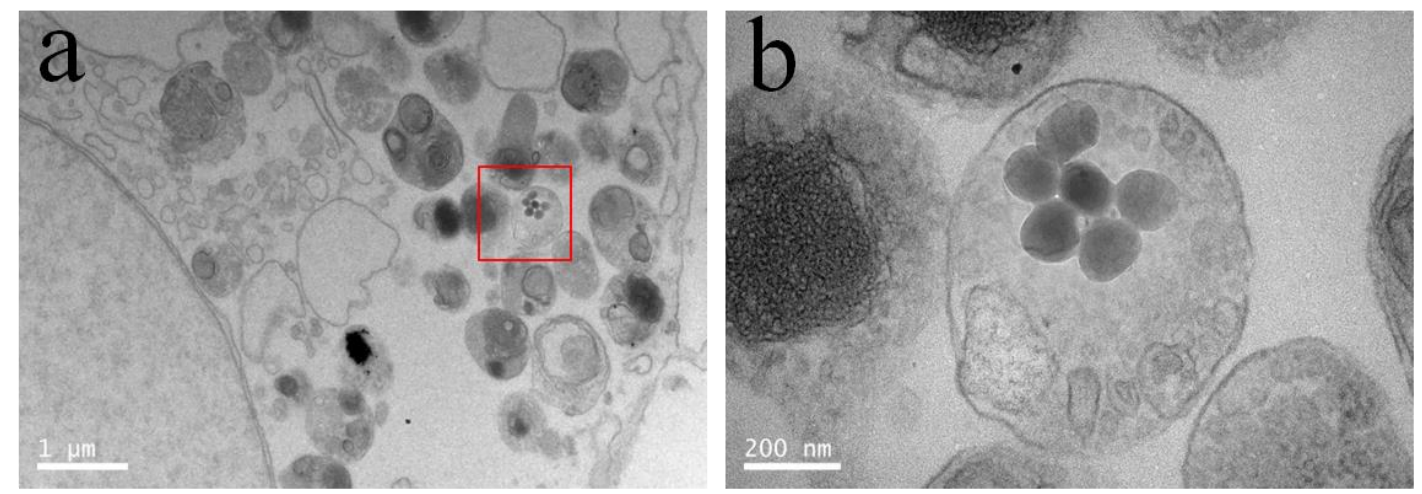

Figure S8. TEM images of a HeLa cell after incubation with DOX-RES-CysM-CPPZ NPs in FBS-free culture medium for 12 h. (a) TEM image of a single HeLa cell, (b) high magnification image of the area within the red box in (a). Scale bars: (a) $1 \mu \mathrm{m}$, (b) $200 \mathrm{~nm}$. 
Table S1. The $\mathrm{IC}_{50}(\mu \mathrm{M})$ values of free DOX, free RES, free RES, DOX-CysM-CPPZ NPs and DOX-RES-CysM-CPPZ NPs after incubation with HeLa or HeLa/ADR cells for $24 \mathrm{~h}$

\begin{tabular}{|c|c|c|c|c|}
\hline \multirow{2}{*}{} & \multicolumn{3}{|c|}{$\mathrm{IC}_{50}(\mu \mathrm{M})$} \\
\cline { 2 - 5 } & free & free & DOX-CysM-CPPZ & DOX-RES-CysM-CPPZ \\
& DOX & RES & NPs & NPs \\
\hline HeLa Cells & 1.01 & 67.50 & 1.34 & 0.46 \\
\hline $\begin{array}{c}\text { HeLa/ADR } \\
\text { Cells }\end{array}$ & 10.40 & 122.31 & 6.19 & 0.65 \\
\hline
\end{tabular}

$\mathrm{IC}_{50}$ : the half-maximal inhibitory concentration

\section{REFERENCES}

1. Hou, S.; Chen, S.; Dong, Y.; Gao, S.; Zhu, B.; Lu, Q., Biodegradable cyclomatrix polyphosphazene nanoparticles: A novel Ph-responsive drug self-framed delivery system. ACS Appl. Mater. Interfaces 2018, 10, 25983-25993.

2. Huang, Z.; Chen, S.; Lu, X.; Lu, Q., Water-triggered self-assembly polycondensation for the one-pot synthesis of cyclomatrix polyphosphazene nanoparticles from amino acid ester. Chem. Commun. 2015, 51, 8373-8376.

3. Hou, S.; Chen, S.; Huang Z.; Lu, Q., Dual-responsive polyphosphazene as a common platform for highly efficient drug self-delivery. J. Mater. Chem. B 2019, 7, $4319-4327$ 